\title{
Asfaltos Colombianos como Precursor de Mesofase Carbonacea
}

\author{
Laura Vargas, Lucas Padilla y Juan Barraza-Burgos* \\ Escuela de Ingeniería Química, Universidad del Valle, A.A. 25360 Cali-Colombia \\ (e-mail: laura.vargas@correounivalle.edu.co; lucas.padilla@correounivalle.edu.co; \\ juan.barraza@correounivalle.edu.co
}

${ }^{*}$ Autor a quien debe ser dirigida la correspondencia

Recibido Ago. 18, 2017; Aceptado Oct. 16, 2017; Versión final Nov. 16, 2017, Publicado Abr. 2018

\section{Resumen}

Se usaron dos tipos de asfaltos colombianos con el fin de determinar su capacidad como un precursor de mesofase de materiales carbonosos. Para la obtención de la mesofase, los asfaltos se sometieron a un tratamiento térmico en un reactor por lotes de acero inoxidable con agitación de $28 \mathrm{rpm}$, a presión atmosférica y bajo una atmosfera inerte generada por un flujo de nitrógeno de $40 \mathrm{~L} / \mathrm{min}$. Para cada asfalto se usó un diseño factorial $2^{2}$ con tres puntos centrales en el que las variables controlables fueron la temperatura de carbonización y el tiempo de residencia. El contenido de mesofase fue la variable de respuesta. Las temperaturas usadas fueron 420 y $460^{\circ} \mathrm{C}$, mientras que los tiempos de residencia fueron 90 y $210 \mathrm{~min}$. Los resultados mostraron que los asfaltos produjeron concentraciones de mesofase en el rango de $50-88 \%$ v/v usando bajos tiempo de residencia y alta temperatura de carbonización.

\section{Colombian Asphalts as a Precursor of Carbonaceous Mesophase}

\begin{abstract}
Two Colombian asphalts were used to determine their capacity as a precursor of mesophase carbonaceous materials. In order to obtain the mesophase, asphalts were carbonized in a stainless steel batch reactor using agitation of $28 \mathrm{rpm}$, atmospheric pressure and inert atmosphere with a nitrogen flow of $40 \mathrm{~L} / \mathrm{min}$. A factorial design $2^{2}$ was used for each coal with three central points. Controllable variables were the carbonization temperature and residence time. The response variable was the mesophase content. The temperatures used were 420 and $460{ }^{\circ} \mathrm{C}$, whereas the residence times were 90 and $210 \mathrm{~min}$. The results showed that asphalts produced mesophase concentrations in the range of $50-88 \% \mathrm{v} / \mathrm{v}$ using low residence time and high carbonization temperature.
\end{abstract}

Keywords: asphalts; carbonization; mesophase; heat treatment; reaction mechanism 


\section{INTRODUCCIÓN}

La producción promedio fiscalizada de petróleo en Colombia fue de 899 Miles de Barriles por Día (KBPD) en el año 2016 (Agencia Nacional de Hidrocarburos, 2016). Estos crudos provenientes de diferentes pozos son en su mayoría pesados (menores a $20^{\circ} \mathrm{API}$ ) y poseen altos porcentajes de asfáltenos (Ecopetrol, 2014). Los asfáltenos se extraen del petróleo con solventes con el fin de facilitar el transporte de este último hacia las diferentes refinerías. Este residuo pesado junto con los asfaltos obtenidos de la refinación del petróleo se usa en su mayoría (cerca del 90\%) para la construcción y pavimentación de vías y carreteras (Ecopetrol, 1999). Diversos trabajos han mostrado el potencial de estos asfáltenos para convertirse en precursor de materiales carbonosos de alta tecnología, entre los cuales se encuentran las fibras de carbono (Mora et. al, 2003), electrodos de grafito (Diefendorf y Riggs, 1980), espumas de grafito (Ji-Hyun et.al. 2016), compuestos de carbón activados (Yue y Vakili, 2017), compuestos de fibra de carbono reforzados con nitrito de boro/resina epóxica (Fan et. al, 2017), nanotubos de carbón (Basante-Romo et.al 2016), entre otros. Para la obtención de dichos precursores los asfáltenos se someten a un proceso de carbonización o polimerización, donde se efectúa un tratamiento térmico bajo una atmosfera inerte por un tiempo determinado.

El precursor obtenido representa un cristal líquido denominado mesofase carbonosa, el cual se obtiene durante el proceso de carbonización del asfalto. Este material, posee características anisotrópicas y puede identificarse a través de un análisis microscópico con luz polarizada (Eser y Jenkins, 1989). Varios estudios han examinado la producción de mesofase a partir de algunos materiales carbonosos (Matsumoto, 1985; Barraza-Burgos y Ospina-Espinosa, 2012; Barraza et. al, 2014). Sin embargo, no se conoce la realización de estudios relacionados que usen asfaltos Colombianos a partir de crudos como material precursor en la producción de mesofase de carbón. El objetivo del trabajo fue evaluar el potencial de dos asfaltos Colombianos como materia prima precursor en la producción de mesofase carbonacea. Se evaluó el efecto de la temperatura y el tiempo de residencia sobre la obtención de la mesofase y se determinaron las mejores condiciones de operación que produjeron el mayor contenido de mesofase.

\section{EXPERIMENTAL}

En esta sección se presentan los materiales y equipos, el software de análisis de imágenes de mesofase, la caracterización de los asfaltos y sus carbonizados, el procedimiento experimental del proceso de carbonización de asfaltos y el análisis microscópico de la mesofase en carbonizados.

\section{Materiales}

Dos tipos de Asfaltos correspondientes a penetración 60/70 1/10 mm (60/70) y 80/100 1/10 mm (80/100) se recibieron de la Empresa Ecopetrol y la Empresa MPI (Barrancabermeja-Colombia) respectivamente. Para la preparación de las probetas de las asfaltos carbonizados se empleó resina líquida epóxica a base de bisfenol a con endurecedor (comercialmente conocidas como "Gemelos A y B") suministradas por el Laboratorio Resiquim, Cali-Colombia. Para el pulido de las probetas se emplearon suspensiones de alúmina de 1.00, 0.30 y 0.05 micras, marca Bühler.

\section{Equipos}

Las corridas de carbonización se realizaron en un reactor por lotes de marca Parr de acero inoxidable 316, de $500 \mathrm{~mL}$, teniendo un agitador de aspas inclinadas, un sistema de enfriamiento para el agitador, un termoposo, válvula de entrada de gas, válvula de muestreo de líquidos, válvula de liberación de gas, disco de ruptura de seguridad y un medidor de presión. El reactor también presenta un controlador de temperatura, referencia 4848 de $115 \mathrm{VAC}$ que opera en un rango de $0-800^{\circ} \mathrm{C}$, el cual cuenta con una termocupla tipo $\mathrm{J}$ de $1 / 8$, un interruptor para el motor y una perilla para el control de la velocidad en el panel frontal.

Con el fin de eliminar las rayas finas producidas en la operación de desbastado y conseguir una superficie lisa de alto brillo en las probetas se empleó la máquina Labopol-5 de marca Struers, en la cual se usan discos adhesivos de marca Carbimet 1-600 PSA/LOO y paños de pulido de marca LECO. Para el análisis de microscopía de luz polarizada de las muestras de los asfaltos carbonizados se utilizó un microscopio metalográfico Eclipse LVD 100 marca Nikon, que produce imágenes con dos tipos de luces: luz reflejada (luz blanca) y luz fluorescente (luz azul). Para este estudio se trabajó sólo con luz blanca y con polarizadores cruzados, empleando un objetivo de 50x para la captura de la imagen.

\section{Software de análisis de imagen}

Para la captura y edición de la imagen se empleó el software LUCIA G versión 5.3 desarrollado por el Laboratorio de Imagen de la Corporación Nikon GmbH. Para el conteo de los puntos efectivos, se superpuso en cada foto una plantilla en forma de malla, con 6 líneas verticales y 4 horizontales. Las 24 intersecciones formadas correspondieron a los puntos a analizar de cada fotografía. 


\section{Caracterización de los asfaltos y carbonizados}

Inicialmente se determinó el punto de ablandamiento de cada uno de los asfaltos, realizado por la empresa MPI, de acuerdo a la norma I.N.V.E-712. Dado que el punto de ablandamiento no es una propiedad fundamental de los asfaltos, al considerar que otro asfalto proveniente de la misma refinería podría tener el mismo valor de penetración, se determinó también caracterizar ambos asfaltos a través de análisis próximo, análisis último y por espectroscopía de resonancia magnética nuclear (H-NMR). Con el objetivo de determinar el contenido de carbono, hidrógeno, nitrógeno y azufre, se realizó un análisis elemental a los asfaltos de petróleo empleando $1 \mathrm{~g}$ de muestra para el análisis. De igual forma, se caracterizaron los productos de la carbonización para cada una de las muestras. Estos análisis se hicieron acorde a la norma ASTM D 5373 para el análisis de C, H, N, y a la norma ASTM D 4239 para el análisis de S.

El análisis próximo (contenido de humedad, ceniza, materia volátil y carbono fijo) de los asfaltos de petróleo y sus carbonizados correspondientes, se realizó acorde a la norma ASTM 7582. También se realizó un análisis cuantitativo de espectroscopía de resonancia magnética nuclear (H-NMR), con el fin de determinar el porcentaje de protones de hidrógeno aromático presentes en los asfaltos. Los diferentes tipos de protones se identificaron en el espectro en los siguientes rangos (Eser y Jenkins, 1989a): protones aromáticos, $9.30-$ $6.30 \mathrm{ppm}$; protones $\alpha, 4.50-1.85$ ppm; protones en los grupos metileno $\beta$ o lejos de los anillos aromáticos y en grupos metilo $\beta$ en los anillos, $1.85-1.00$ ppm; protones en el grupo terminales metilo y o más lejanos de los anillos. La distribución de los átomos de hidrógeno constituyentes entre grupos aromáticos y alifáticos se determinó por medio de la integración digital de las intensidades de bandas normalizadas a lo largo del espectro completo $(9.30-0.50 \mathrm{ppm})$.

\section{Proceso de carbonización de asfaltos}

El diseño experimental usado incluyó la temperatura de carbonización y el tiempo de residencia como variables de control. Para cada carbón, se utilizó un diseño factorial $2^{2}$ con dos niveles para cada variable y se establecieron 3 puntos centrales para evaluar la repetibilidad de los experimentos. La variable de respuesta fue el contenido de mesofase (\% v/v) obtenido en cada corrida. La Tabla 1 muestra la configuración de cada una de las 14 corridas realizadas especificando el tipo de asfalto y los niveles de cada variable.

Tabla 1: Tipos de Asfalto, tiempo de residencia y temperatura de operación usadas en la carbonización

\begin{tabular}{|c|c|c|c|}
\hline Prueba & Asfalto & Tiempo (min) & Temperatura $\left({ }^{\circ} \mathrm{C}\right)$ \\
\hline$A(-1,1)$ & \multirow{7}{*}{$60 / 70$} & 90 & 420 \\
\hline$A(-1,1)$ & & 90 & 460 \\
\hline $\mathrm{A}(0,0) 1$ & & 150 & 440 \\
\hline$A(0,0) 2$ & & 150 & 440 \\
\hline $\mathrm{A}(0,0) 3$ & & 150 & 440 \\
\hline$A(1,-1)$ & & 210 & 420 \\
\hline$A(1,1)$ & & 210 & 460 \\
\hline$B(-1.1)$ & \multirow{7}{*}{$80 / 100$} & 90 & 420 \\
\hline$B(-1,1)$ & & 90 & 460 \\
\hline $\mathrm{B}(0,0) 1$ & & 150 & 440 \\
\hline $\mathrm{B}(0,0) 2$ & & 150 & 440 \\
\hline $\mathrm{B}(0,0) 3$ & & 150 & 440 \\
\hline$B(1,-1)$ & & 210 & 420 \\
\hline $\mathrm{B}(1,1)$ & & 210 & 460 \\
\hline
\end{tabular}

La velocidad de agitación mecánica se estableció en 28 rpm además de la agitación producida por las burbujas de nitrógeno. Se estableció un flujo de nitrógeno de $40 \mathrm{~L} / \mathrm{h}$. Mora et.al, 2003 realizaron experimentos con un reactor similar al usado en este trabajo, por lo cual este valor se ajustó a las características del proceso. Una vez cargado el reactor con los asfaltos se realizó una purga con nitrógeno burbujeado durante 5 minutos. Posteriormente, se montó el reactor en la zona de calentamiento y se ajustó la temperatura de reacción requerida, dejando reaccionar el sistema durante el tiempo estipulado. Finalizada la reacción, se realizó un enfriamiento súbito (quenching). Finalmente, se retiraron los productos del reactor, se pesaron y se guardaron para análisis físico-químico y microscópico. 


\section{Análisis microscópico de la mesofase en carbonizados}

El análisis microscópico de la mesofase de carbono se realizó según la norma ASTM D4616 - 95. Las muestras obtenidas de la carbonización se prepararon para su posterior análisis de la mesofase producida en el microscopio. La resina epóxica donde se embebió la muestra carbonizada se preparó en una mezcla de proporción $50 \%$ p/p resina y $50 \%$ p/p de endurecedor y se mezcló hasta obtener buena homogeneidad. Luego, $4 \mathrm{~g}$. de resina se mezclaron con $2 \mathrm{~g}$ de asfalto carbonizado a analizar hasta obtener una mezcla uniforme, la cual se llevó a un sistema de vacío con el fin de extraer las burbujas presentes. La probeta, una vez estuvo completamente seca y dura, se devastó con lijas 400, 600 y 1200. Finalmente se realizó el pulimento con máquina y paños usando soluciones de alúminas de $1.00,0.50$ y 0.05 micras. El asfalto carbonizado se analizó en el microscopio para identificar y contar la mesofase producida. Mayores detalles de este análisis de imágenes se presentan en el trabajo de Barraza-Burgos y Ospina-Espinosa, 2012.

\section{RESULTADOS Y DISCUSION}

En esta sección se presentan los resultados y la discusión de los resultados relacionados con la caracterización de los asfaltos originales y sus carbonizados, los rendimientos másicos de la carbonización, Identificación y cuantificación de la mesofase, los tipos de morfología y los mecanismos propuestos de obtención de las mesofases.

\section{Caracterización de los asfaltos originales}

Los dos asfaltos usados se caracterizaron de acuerdo a los análisis mostrados en la Tabla 2. Se observó que ambos asfaltos presentaron alto contenido de materia volátil y son ricos en carbono. También tienen baja aromaticidad (bajo contenido de protones aromáticos Har), al compararlos con asfaltos utilizados en la literatura (Eser y Jenkins, 1989a; Santamaría-Ramírez et. al., 1989). De igual forma exhiben un bajo contenido de ceniza, humedad y heteroatomos de $\mathrm{S}$ y $\mathrm{N}$.

Tabla 2: Caracterización de los asfaltos de petróleo originales

\begin{tabular}{|c|c|c|c|}
\hline & Norma & Asfalto $60 / 70$ & Asfalto $80 / 100$ \\
\hline Punto de ablandamiento $\left({ }^{\circ} \mathrm{C}\right)$ & I.N.V.E-712 & 49.0 & 46.4 \\
\hline \multicolumn{4}{|l|}{ Análisis próximo (\%p/p) } \\
\hline Ceniza & ASTM D 7582 & 0.02 & 0.03 \\
\hline Materia volátil & ASTM D 7582 & 91.26 & 91.75 \\
\hline Humedad total & ASTM D 3302 & 0.16 & 0.14 \\
\hline Carbono fijo & Por diferencia & 8.58 & 8.08 \\
\hline \multicolumn{4}{|l|}{ Análisis último (\%p/p) } \\
\hline $\mathrm{C}$ & ASTM D 5373 & 86.32 & 88.04 \\
\hline $\mathrm{H}$ & ASTM D 5373 & 10.56 & 10.24 \\
\hline$N$ & ASTM D 5373 & 0.64 & 0.22 \\
\hline$S$ & ASTM D 4239 & 1.86 & 1.40 \\
\hline $\mathrm{O}$ & Por diferencia & 0.62 & 0.10 \\
\hline $\mathrm{C} / \mathrm{H}$ & & 0.69 & 0.72 \\
\hline H-NMR, Har (\%) & & 7.69 & 11.20 \\
\hline
\end{tabular}

Con respecto a los puntos de ablandamiento presentados por los asfaltos, se observa que existe alguna diferencia posiblemente debido a los contenidos de materia volátil presente en cada asfalto. Conforme se disminuye el contenido de materia volátil, el punto de ablandamiento aumenta haciendo los asfaltos menos fluidos. Lo anterior se confirma dado que el Asfalto 80/100 presenta un mayor contenido de materia volátil $(91.75 \% \mathrm{p} / \mathrm{p})$, y por ende un menor punto de ablandamiento $\left(46.4^{\circ} \mathrm{C}\right)$, mientras que el Asfalto $60 / 70$ presenta el caso contrario, $91.26 \% \mathrm{p} / \mathrm{p}$ y $49.0^{\circ} \mathrm{C}$ respectivamente. Esos resultados son consistentes con los reportados por Huang, 2009. De acuerdo a los espectros H-NMR obtenidos para los Asfaltos 80/100 y 60/70 presentados en las figuras 1 y 2 respectivamente, así como a los valores obtenidos en la integración digital de las intensidades de bandas normalizadas, se calculó el porcentaje de protones de hidrógeno aromáticos presentes en cada asfalto. El Asfalto $80 / 100$ presentó un mayor grado de aromaticidad (11.20\%) que el Asfalto 60/70 (7.69\%). Esta aromaticidad, puede relacionarse con los contenidos de materia volátil, relación C/H y puntos de ablandamiento obtenidos. Eser y Jenkins, 1989 encontraron una relación directa entre la aromaticidad, la razón $\mathrm{C} / \mathrm{H}$ y el contenido de materia volátil. Sin embargo, también obtuvieron una relación inversa entre la aromaticidad y el punto de ablandamiento. 


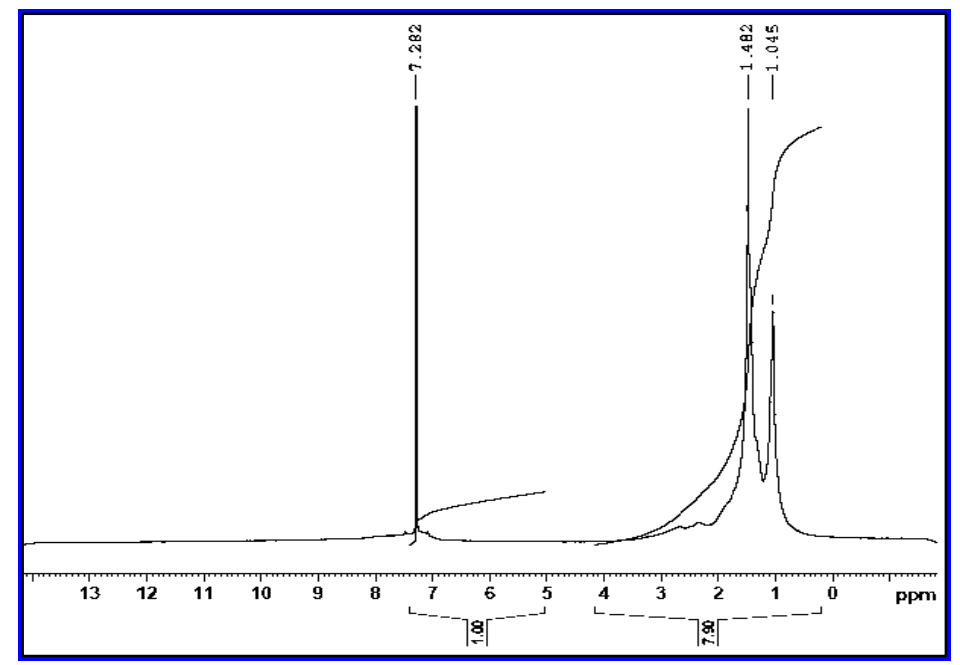

Fig. 1: Espectro H-NMR del asfalto 80/100

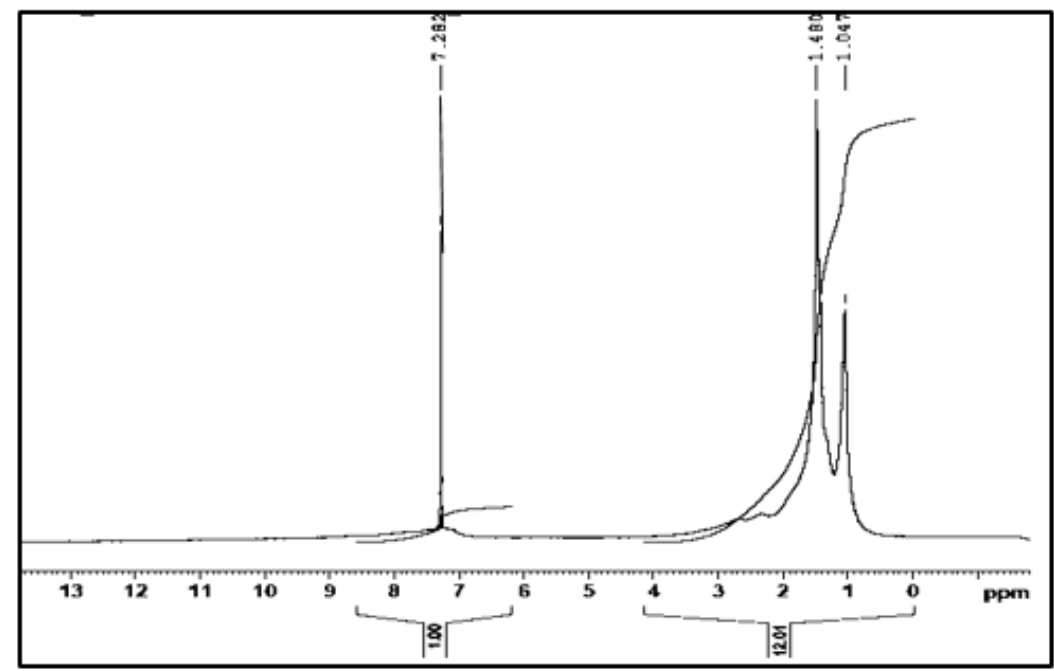

Fig. 2: Espectro H-NMR del asfalto 60/70

El contenido relativamente bajo de $\mathrm{S}$ y $\mathrm{N}$ de ambos asfaltos puede atribuirse al bajo contenido de heteroatomos de sus precursores, y al proceso de ruptura que tuvieron durante su producción (Eser y Jenkins, 1989). Se conoce que los heteroatomos de $S$ retardan el desarrollo de la mesofase (Eser y Jenkins, 1989a). Las cenizas representan el contenido de sólidos no fundibles presentes en los asfaltos (Matsumoto, 1985). La presencia de estos sólidos durante la carbonización altera la interface, la estructura y la nucleación de la mesofase (Mora et. al, 2003; Korai et. al., 1997; Mora et. al., 2003). Ambos asfaltos tienen un bajo contenido de ceniza, lo cual las podría hacer aptas para la producción de fibras de carbono debido a que se facilita su estabilidad durante el hilado. El bajo contenido de aromáticos sugiere un bajo rendimiento en la cantidad de mesofase obtenida para ambas asfaltos, tal como también fue reportado por Matsumoto (1985).

\section{Caracterización de los asfaltos carbonizados}

Los asfaltos carbonizados se caracterizaron de acuerdo a los análisis mostrados en la Tabla 3 . En este trabajo se analizó el asfalto carbonizado obtenido en las pruebas $A(0,0) 2$ y $B(0,0) 2$, las cuales definieron la repetibilidad de los experimentos. Tal como se observa en la Tabla 3, se produjo una reducción del contenido de materia volátil y del aumento de la razón $\mathrm{C} / \mathrm{H}$ en los asfaltos carbonizados en comparación con los asfaltos originales. Similares resultados se encontraron en los trabajos de Huang, 2009; Blanco et. al, 2000, quienes demostraron que las condiciones de las reacciones de carbonización deshidrogenativas que ocurren como condensaciones aromáticas, así como la pérdida de las moléculas más pequeñas que contienen hidrógenos alifáticos, explican este comportamiento. También en la tabla 3 se observa que el porcentaje de ceniza aumentó en los asfaltos carbonizados en comparación con los asfaltos originales (Tabla 2). Lo anterior lo causó la cantidad de gases condensables e incondensables liberados. 
Tabla 3: Caracterización de los asfaltos carbonizados

\begin{tabular}{|c|c|c|c|}
\hline & Norma & Asfalto $60 / 70$ & Asfalto $80 / 100$ \\
\hline \multicolumn{4}{|c|}{ Análisis próximo (\% p/p) } \\
\hline Ceniza & ASTM D 7582 & 0.53 & 0.77 \\
\hline Materia volátil & ASTM D 7582 & 10.91 & 12.72 \\
\hline Humedad total & ASTM D 3302 & 0.69 & 1.94 \\
\hline Carbono fijo & Por diferencia & 87.87 & 84.57 \\
\hline \multicolumn{4}{|c|}{ Análisis último(\% p/p) } \\
\hline C & ASTM D 5373 & 89.97 & 88.37 \\
\hline $\mathrm{H}$ & ASTM D 5373 & 4.03 & 3.64 \\
\hline $\mathrm{N}$ & ASTM D 5373 & 2.11 & 2.91 \\
\hline S & ASTM D 4239 & 2.43 & 2.43 \\
\hline $\mathrm{O}$ & Por diferencia & 1.46 & 2.65 \\
\hline $\mathrm{C} / \mathrm{H}$ & & 1.87 & 1.93 \\
\hline
\end{tabular}

\section{Rendimientos másicos de asfaltos carbonizados}

La Tabla 4 muestra los rendimientos obtenidos de los asfaltos carbonizados, gases condensados y volátiles no condensables, a diferentes condiciones de temperatura y tiempo de carbonización. Se observa que el rendimiento másico para cada reacción lo afecta la temperatura y el tiempo de reacción. Los resultados muestran que las corridas para las condiciones bajas de operación de $420^{\circ} \mathrm{C}$ y $90 \mathrm{~min}$, se produjeron altos rendimientos másicos de $29.98 \%$ para el Asfalto $80 / 100$ y de $24.67 \%$ para el Asfalto $60 / 70$ respectivamente. Caso contrario existió en los niveles de operación de $460^{\circ} \mathrm{C}$ y $210 \mathrm{~min}$, donde se encontraron menores rendimientos. Éste comportamiento se esperaba para el proceso, ya que conforme aumenta la temperatura se retira más material del sistema como condensados o como materia volátil. De la misma forma, altos tiempos de reacción favorece el retiro de materia volátil, por lo cual el rendimiento másico decrece.

Tabla 4: Rendimiento másico de carbonizados y concentración de mesofase en los carbonizados

\begin{tabular}{|c|c|c|c|c|c|}
\hline Prueba & Asfalto & $\begin{array}{l}\text { Tiempo } \\
\text { (min) }\end{array}$ & $\begin{array}{c}\text { Temperatura } \\
\left({ }^{\circ} \mathrm{C}\right) \\
\end{array}$ & $\begin{array}{c}\text { Rendimiento } \\
(\% p / p)\end{array}$ & $\begin{array}{c}\text { Mesofase } \\
(\% \mathrm{~V} / \mathrm{v})\end{array}$ \\
\hline$A(-1,1)$ & & 90 & 420 & 24.67 & 50.20 \\
\hline$A(-1,1)$ & & 90 & 460 & 23.40 & 84.62 \\
\hline$A(0,0) 1$ & & 150 & 440 & 25.40 & 77.62 \\
\hline$A(0,0) 2$ & $60 / 70$ & 150 & 440 & 19.87 & 82.63 \\
\hline$A(0,0) 3$ & & 150 & 440 & 21.42 & 74.00 \\
\hline$A(1,-1)$ & & 210 & 420 & 22.02 & 86.01 \\
\hline$A(1,1)$ & & 210 & 460 & 22.96 & 86.71 \\
\hline$B(-1.1)$ & & 90 & 420 & 29.98 & 58.77 \\
\hline $\mathrm{B}(-1,1)$ & & 90 & 460 & 25.23 & 87.15 \\
\hline $\mathrm{B}(0,0) 1$ & & 150 & 440 & 24.40 & 77.46 \\
\hline$B(0,0) 2$ & $80 / 100$ & 150 & 440 & 24.19 & 80.27 \\
\hline $\mathrm{B}(0,0) 3$ & & 150 & 440 & 19.14 & 82.52 \\
\hline$B(1,-1)$ & & 210 & 420 & 22.23 & 85.77 \\
\hline $\mathrm{B}(1,1)$ & & 210 & 460 & 21.39 & 88.43 \\
\hline
\end{tabular}

Los asfaltos de petróleo están constituidos principalmente por cuatro grupos de sustancias: saturados, naftenos aromáticos (mono-di-aromáticos), aromáticos polares (resinas) y asfáltenos. Los rendimientos obtenidos durante el proceso experimental muestran que la mesofase se forma a partir de las fracciones más pesadas del asfalto (asfáltenos y compuestos aromáticos polares), mientras que la material volátil y los condensados obtenidos corresponden a compuestos de menor peso molecular (Brooks et. al., 1965). Lo anterior está acorde con los resultados del contenido de material volátil de ambas asfaltos. De la Tabla 2, se observa que la materia volátil fue de $91.26 \%$ para el Asfalto $60 / 70$ y de $91.75 \%$ para el Asfalto 80/100. 
Asimismo, el contenido de aromáticos presentes fue de $8.61 \%$ y $8.08 \%$ respectivamente, representando la fracción más ligera del asfalto. Por lo anterior, gran cantidad de volátiles se retira, y por ende podría considerarse que el material carbonizado está compuesto de asfáltenos y de aromáticos polares.

\section{Identificación y cuantificación de la mesofase}

Para cuantificar la producción de mesofase en los asfaltos carbonizados fue necesario conocer su correcta identificación. En la Figura 3, se identifican cada una de las especies consideradas durante la realización del conteo de puntos efectivos. Durante el conteo únicamente se tuvo en cuenta los puntos situados en partículas enfocadas donde se identificaron las fases isotrópicas y anisotrópicas. Sí el punto se situaba sobre una resina, un agujero causado por la porosidad de la partícula o sobre alguna parte desenfocada, no se consideraba para el conteo. Teniendo en cuenta el efecto del cambio del ángulo de incidencia de la luz sobre la anisotropía se capturaron dos fotografías para cada imagen enfocada, ambas con ángulos de incidencia diferentes. Al modificar dicho ángulo se evidenció que la fase isotrópica permanece sin cambios, mientras que la fase anisotrópica reflejó la luz en una dirección diferente, como se observa en la Figura 3. En la Tabla 4 también se muestra el porcentaje de mesofase obtenido para cada prueba. La mayor producción de mesofase obtenida a $210 \mathrm{~min}$ y $460{ }^{\circ} \mathrm{C}$ fue de $88.43 \%(\mathrm{v} / \mathrm{v})$, mientras que la menor producción obtenida 90 min y 420 ${ }^{\circ} \mathrm{C}$ fue de $50.2 \%(\mathrm{v} / \mathrm{v})$. Lo anterior indica que el rendimiento de producción de mesofase lo afectaron la temperatura y el tiempo de residencia del proceso de carbonización.

\section{Tipos de morfología y mecanismo de mesofase obtenidas}

Las mesofases carbonosas obtenidas después del proceso de carbonización presentan diferentes texturas. La morfología o textura adoptada por la mesofase depende de las condiciones de carbonización y naturaleza química de los materiales (Braga et.al., 2009). En la Figura 4 se presentan las morfologías obtenidas para cada corrida efectuada con el Asfalto 80/100. En general, se observan diferentes tipos de texturas producidas, posiblemente asociadas a los efectos de agitación, burbujeo del nitrógeno o naturaleza química de los asfaltos de petróleo utilizados. Se observó el mismo comportamiento y las mismas morfologías en las pruebas realizadas con el Asfalto 60/70.

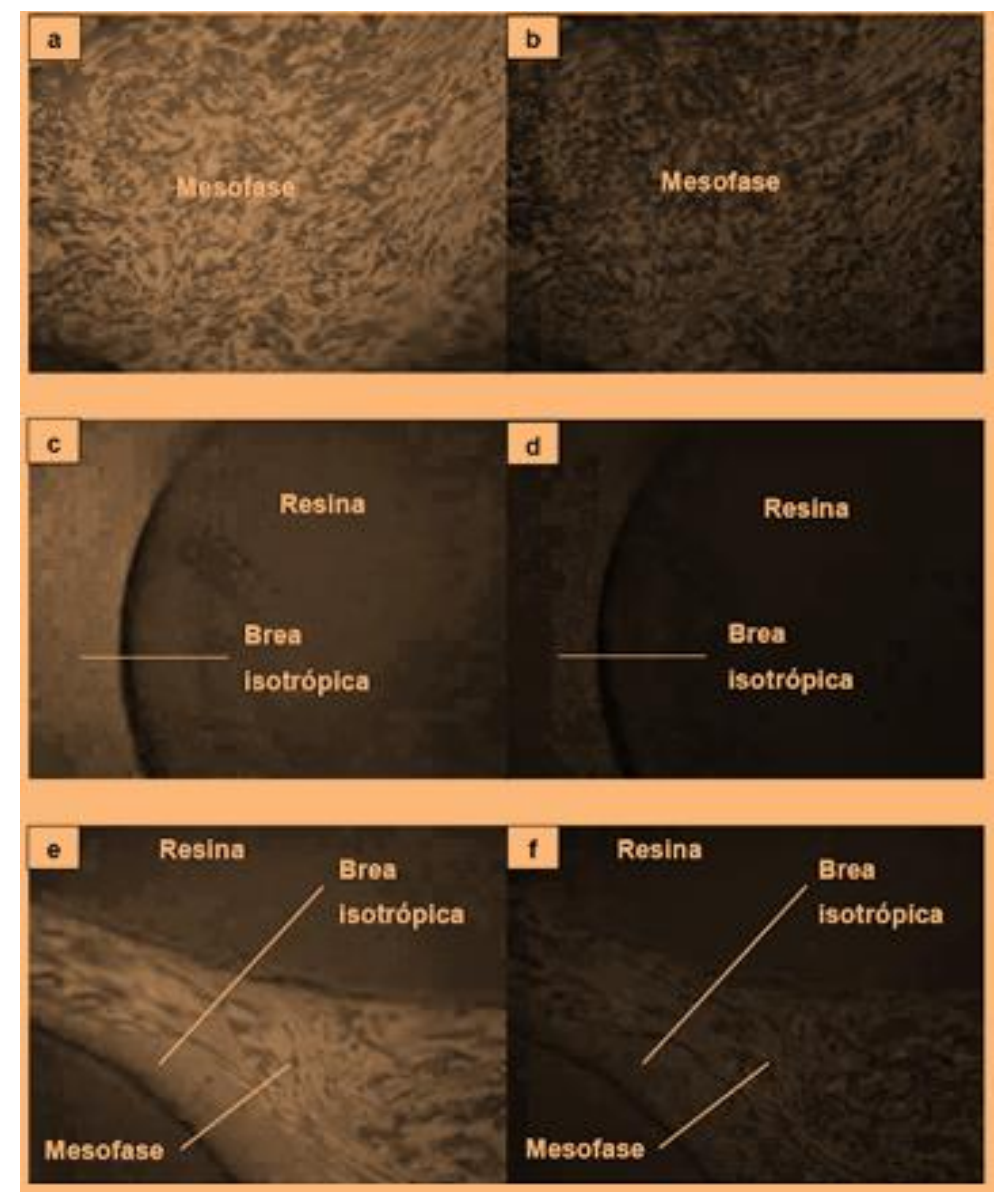

Fig. 3: Identificación de la mesofase, fase anisotrópica y resina. Muestra de la corrida $\mathrm{B}(-1,-1)$. Partícula con sólo mesofase: (a) $180^{\circ}$, (b) $225^{\circ}$. Partícula anisotrópica: (c) $180^{\circ}$, (d) $225^{\circ}$. Partícula con mesofase y fase anisotrópica; (e) $180^{\circ}$, (f) $225^{\circ}$ 


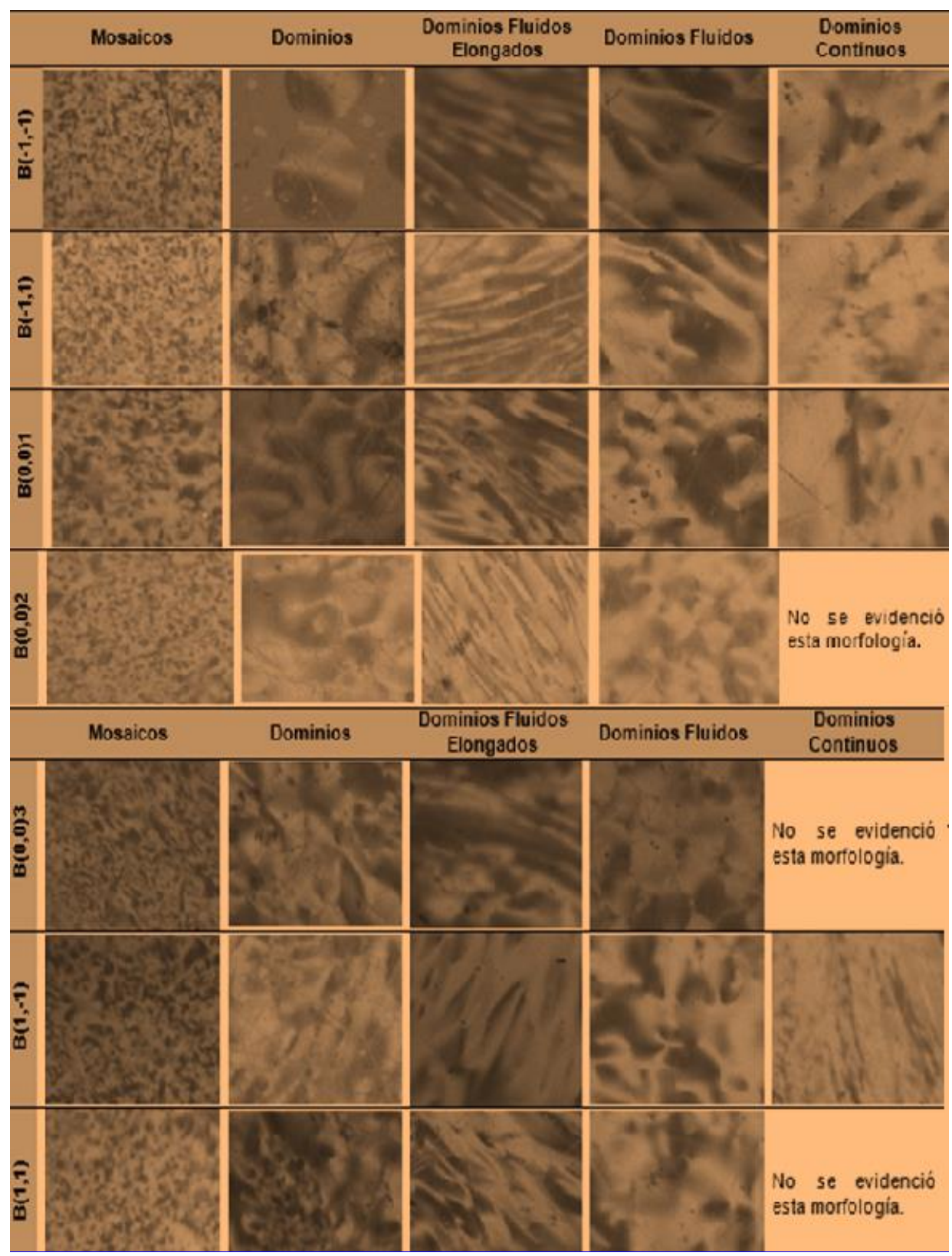

Fig. 4: Morfologías obtenidas en las pruebas realizadas con el Asfalto 80/100

Marsh et. al. 1999 propusieron que el mecanismo de producción de la morfología de la mesofase se lleva a cabo cuando los mesógenos formados por la condensación de los compuestos aromáticos se asocian. Un mesógeno existe como una sola molécula. Sin embargo, cuando se asocia con otro, ambos hacen parte de la fase anisotrópica (mesofase). Normalmente, existen dos formas de asociación entre los mesógenos: en la primera establecen una nueva unidad de crecimiento del cristal líquido, y en la segunda, se asocian con unidades de crecimiento ya establecidas. Este es un proceso aleatorio relacionado con las interacciones de las moléculas en el líquido isotrópico. En el presente trabajo, la agitación y el burbujeo del nitrógeno, además de mejorar la transferencia de energía y la eliminación de la material volátil, podrían promover la coalescencia de los mesógenos formados.

La formación del mosaico (segunda columna en la Figura 4) inició con la coalescencia de dos esferas. De forma general, estas dos esferas tienen diferente orientación. Cuando estas chocan la superficie de ambas se reorganizan para permitir la unión de estas. Estas interacciones continúan en todas las dimensiones y tras un periodo de tiempo se forma una estructura denominada "mosaico" donde apenas se distinguen las formas esféricas iniciales. Las morfologías de mosaico encontrada en este estudio son consistentes con la presentada por Brooks et. al., 1965. La generación de dominios en mosaico posiblemente se relaciona con la presencia de partículas sólidas presentes en el asfalto (en este caso ceniza), que generan una dificultad de las esferas de mesofase para coalescer. Los resultados obtenidos también muestran que estos sólidos no solo controlan la coalescencia de las esferas mesofase, también controlan la nucleación de las esferas. Esos resultados están de acuerdo con los encontrados por Mora, 2003. Brookst. al, 1965 encontraron que para la 
elaboración de fibras de carbono a partir de mesofase carbonosa, es conveniente tener una concentración de sólidos (ceniza) menor del $0.1 \%$ en el asfalto sin tratar. En este trabajo, se encontró que la morfología predominante no fue de tipo mosaico por la baja concentración de sólidos presentes en los asfaltos (ver Tabla 2). Por lo anterior, estos resultados sugieren que los asfaltos usados podrían representan un potencial precursor para la producción de fibras de carbono.

Por otro lado, en la ausencia de sólidos no fundibles, también se observó (tercera columna en la Figura 4) la coalescencia de esferas de mayor tamaño, evidenciándose la fase isotrópica. Lo anterior sugiere que la mayor parte del material se convirtió en mesofase con grandes zonas de dominios fluidos elongados causados por el efecto de la agitación, así como también, dominios continuos que tiene una orientación casi constante y homogénea. Esto ocurrió principalmente (cuarta, quinta y sexta columnas en la Figura 4) a causa de que la mesofase estuvo libre de asfalto sin convertir. Similares encuentros se obtuvieron en el trabajo de Brooks et. al, 1965. Los resultados mostraron que al incrementar la temperatura de reacción y/o el tiempo de residencia se presentó mayor coalescencia y existió la tendencia a predominar las morfologías de dominios fluidos y dominios fluidos elongados. Los dominios continuos se presentan pero no se observa que predominen.

\section{CONCLUSIONES}

A partir de dos asfaltos de petróleo Colombianos 60/70 y 80/100 se obtuvo mesofase carbonosa. El tipo de asfalto no tuvo un impacto significativo en la producción de mesofase, debido a la similitud entre ambos. Se determinó que la temperatura y el tiempo de reacción tuvieron influencia en el proceso. El porcentaje de mesofase aumentó conforme se incrementó el valor de cada variable hasta el nivel máximo establecido (210 minutos y $460^{\circ} \mathrm{C}$ respectivamente), obteniendo conversiones de 88.43 y $86.01 \% \mathrm{v} / \mathrm{v}$ para las pruebas $\mathrm{B}(1,1)$ y $A(1,1)$ respectivamente.

Para cada asfalto, las mejores condiciones del proceso fueron un tiempo de residencia de 90 minutos, y temperatura de carbonización de $460^{\circ} \mathrm{C}$. Un tiempo menor mejoró la productividad del proceso, asimismo una alta temperatura aumentó las interacciones moleculares que aceleraron el proceso de producción de mesofase carbonosa. La mesofase carbonosa se obtuvo de la fracción más pesada de los asfaltos y no se observó una única morfología. Lo anterior muestra la versatilidad del asfalto como posible precursor de mesofase para obtener diferentes tipos de materiales carbonosos. De acuerdo a la aplicación a la que se desee destinar la mesofase, el proceso de carbonización y la naturaleza química del asfalto pueden modificarse con el fin de obtener la morfología requerida como predominante. Debido al bajo contenido de sólidos no fundibles presentes en los asfaltos, pueden considerarse como un precursor potencial para la producción de fibras de carbono.

\section{AGRADECIMIENTOS}

Los autores agradecen el apoyo de las Empresas Ecopetrol e MPI (Barrancabermeja-Colombia) por el suministro de muestras de asfaltos utilizados en el presente estudio y por el análisis del punto de ablandamiento de los asfaltos.

\section{REFERENCIAS}

Agencia Nacional de Hidrocarburos. Producción mensual de petróleo. En la web: https://goo.gl/MDM2TL, acceso mayo (2016)

Barraza, J., N. Muñoz y L. Barona; Asphaltenes and preasphaltenes from coal liquid extracts: feedstocks to obtain carbon mesophase, Rev. Fac. Ing. Univ. Antioquia, ISSN: 0120-6230. En línea: https://goo.gl/j7mq7Z. 70, 99-107 (2014)

Barraza-Burgos, J.M. y J.M. Ospina-Espinosa, J.M; Effect of pitch petroleum oxidation on mesophase production. CT\&F - Cie. Tec. y Fut., ISSN 0122-5383. En línea: https://goo.gl/Smg1Dp, 4(5), 33-42 (2012)

Basante-Romo, M., J, Benavides-Guerrero y R. Camargo-Amado; Síntesis y Caracterización del Nanomaterial TiO2MWCNTs (Oxido de Titanio-Nanotubos de Carbono de Pared Múltiple). doi: 10.4067/S0718-07642016000600016, Inf. Tecnol (en línea), 27(6), 153-162 (2016)

Blanco, C., R. Santamaría, y otros dos autores; Comparative study of air-blown and thermally treated coal-tar pitches, https://doi.org/10.1016/S0008-6223(99)00131-1, Carbon (en línea), 38, 517-523 (2000)

Braga, C., C. Dutra, L. y otros dos autores; Influence of heat and pressure treatment on the rheological behavior of petroleum pitches, https://doi.org/10.1016/j.fuel.2008.10.029, Fuel (en línea), 88 (5), 853-860 (2009)

Brooks, J. y, G. Taylor; The formation of graphitizing carbons from the liquid phase, https://doi.org/10.1016/00086223(65)90047-3, Carbon (en línea), 3 (2), 185-186 (1965)

Diefendorf, R. y D. Riggs; Forming Optically Anisotropic Pitches. U.S. Patent US4208267 (1980)

Ecopetrol; Cartilla práctica para el manejo de los asfaltos colombianos. En la web: https://goo.gl/u9wtUp. Acceso: junio 
(1999)

Ecopetrol; Exportaciones de crudo. En la web: https://goo.gl/WW6zBr. Acceso octubre (2017)

Eser, S. y R. Jenkins; Carbonization of petroleum feedstocks I: Relationships between chemical constitution of the feedstocks and mesophase development, https://doi.org/10.1016/0008-6223(89)90038-9, Carbon (en línea), 27 (6), 877887 (1989)

Eser, S. y R. Jenkins; Carbonization of petroleum feedstocks II: Relationships between chemical constitution of the feedstocks and mesophase development, https://doi.org/10.1016/0008-6223(89)90039-0, Carbon (en línea), 27 (6), 889897, (1989a)

Fan, B., Y. Liu, y otros dos autores; Enhanced thermal conductivity for mesophase pitch-based carbon fiber/modified boron nitride/epoxy composite, https://doi.org/10.1016/j.polymer.2017.06.060, Polymer (en línea), 122 (28), 71-76 (2017)

Huang, H.; Fabrication and properties of carbon fibers, doi:10.3390/ma2042369, Materials, (en línea), 2 (4), 2369-2403 (2009)

Ji-Hyun, K., D. Young, y otros dos autores; Physical-Chemical Properties of Graphite Foams Produced with Fluorinated Mesophase Pitch, doi:10.9713/kcer.2016.54.6.830, Korean Chem. Eng. Res., (en línea), 54, (6), 830-837 (2016)

Korai, Y., S. Ishida, y otros dos autores; Preparation of mesocarbon microbeads by dispersing mesophase pitch in isotropic pitches, https://doi.org/10.1016/S0008-6223(97)00101-2,Carbon, (en línea), 35 (10), 1503-1515 (1997)

Marsh, H., M. Martínez-Escandell y F. Rodríguez-Reinoso; Semicokes from pitch pyrolysis: mechanisms and kinetics, https://doi.org/10.1016/S0008-6223(98)00205-X, Carbon, (en línea), 37, 363-390 (1999)

Matsumoto, T.; Mesophase pitch and its carbon fibers, https://doi.org/10.1351/pac198557111553, Pure Appl. Chem., (en línea), 57 (11), 1553-1562 (1985)

Mora, E., C. Blanco., y otros tres tres autores; A novel method to obtain a petroleum-derived mesophase pitch suitable as carbon fibre precursor, https://doi.org/10.1016/S0008-6223(02)00354-8, Carbon, (en línea), 41 (3), 445-452 (2003)

Mora, E., R. Santamaría, y otros tres tres autores; Mesophase development in petroleum and coal-tar pitches and their blends, https://doi.org/10.1016/S0165-2370(03)00034-2, J. Anal. Appl. Pyrolysis, (en línea), 68-69, 409-424 (2003)

Yue, Z, y A. Vakili. Activated carbon-carbon composites made of pitch-based carbon fibers and phenolic resin for use of adsorbents, doi: 10.1007/s10853-017-1389-7, Journal of Mat. Sci., (en línea), 52 (21), 12913-12921 (2017) 